Acta Sci. Pol. Technol. Aliment. 19(1) 2020, 109-122

pISSN 1644-0730

eISSN 1898-9594

http://dx.doi.org/10.17306/J.AFS.2020.0729

ORIGINAL PAPER

Received: 21.10.2019

Accepted: 30.12.2019

\title{
POLYMORPHISM OF TAS2R3, TAS2R5, TAS2R19, AND TAS2R5O GENES AND BITTER FOOD INTAKE FREQUENCY IN ELDERLY WOMAN
}

\author{
Joanna Mikołajczyk-Stecyna ${ }^{\bowtie}$, Anna M. Malinowska, Agata Chmurzynska \\ Institute of Human Nutrition and Dietetics, Poznan University of Life Sciences \\ Wojska Polskiego 31, 60-624, Poznań, Poland
}

\begin{abstract}
Background. Taste sensitivity is one of the most important biological determinants of food choice. Polymorphisms within the bitter taste receptor genes TAS2R3 (rs765007), TAS2R5 (rs2234012), TAS2R19 (rs10772420), and TAS2R50 (rs1376251) may affect bitter taste sensitivity and thus food choices and thereby metabolic biomarkers in blood. The aim of this study was to investigate associations between selected TAS2Rs single nucleotide polymorphisms (SNPs) and the choice of the most popular bitter food items in a Polish population, BMI and blood biomarkers in elderly women.

Material and methods. The study group included 116 Polish women over 60 years of age. Intake of Brassica vegetables, grapefruit and coffee was assessed using a food frequency questionnaire. Biochemical parameters were measured using the spectrophotometric method. Genotyping was performed using the high resolution melting method.

Results. We show an association between SNPs of the TAS2R3 gene and the frequency of Brassica vegetable intake, between SNPs of the TAS2R5 gene and the frequency of grapefruit intake, and between the simultaneous effects of polymorphisms within TAS $2 R 3$ and TAS2R5 and the frequency of eating Brassica vegetables in general. We found no association between the genetic polymorphisms of TAS2R19 or TAS2R50 that were examined and the frequency of bitter-tasting food intake. Moreover, the SNPs of the selected TAS2Rs genes may be associated with the lipid profile, serum level of glucose and CRP, depending on the frequency of consumption of particular bitter-tasting items.

Conclusions. The genetic polymorphisms analyzed in the study seem not to contribute significantly to variability in bitter-tasting food intake in elderly women, although they may influence metabolic biomarkers dependent on the intake of particular bitter-tasting food items.
\end{abstract}

Keywords: bitter taste, blood lipid biomarkers, consumption frequency, genetic polymorphism, TAS2R3, TAS2R5, TAS2R19, TAS2R50

\section{INTRODUCTION}

Taste sensitivity is the most important biological determinant of food choice (Kourouniotis et al., 2016). A substantial body of evidence indicates that there are differences in taste perception among individuals, which are due in part to the abundant genetic variations in chemoreceptor genes. Because of the large number of different ligands (Meyerhof et al., 2010), bitter taste perception seemed to be the most complex of the 
well-defined tastes in humans. Bitter taste transduction occurs through 25 bitter taste receptors $(T A S 2 R s)$ that are specific to particular classes of ligands (Behrens et al., 2007). Polymorphisms of TAS2R genes may affect the sensation, liking or intake of common food and beverages, i.e. vegetables, fruits or alcohol and coffee (Colares-Bento et al., 2010; Duffy et al., 2010; Sacerdote et al., 2007). These bitter-tasting food products may contain phytochemicals (i.e. isothiocyanates, caffeine, chlorogenic acid, naringin and naringenin), whose intake has been associated with prevention of noncommunicable diseases such as obesity and cardiovascular diseases (Alam et al., 2014; Choi et al., 2012; Dinkova-Kostova, 2013).

One of the first and most widely studied bitter taste receptor genes is TAS2R38. Three single nucleotide polymorphisms (SNPs) within the coding sequence of $T A S 2 R 38$ give rise to two common haplotypes, called PAV and AVI (Kim et al., 2005). Although TAS2R38 haplotype is responsible for interindividual differences in perceiving $\mathrm{N}-\mathrm{C}=\mathrm{S}$ moiety (occurs in the isothiocyanates present in Brassica vegetables (Bufe et al., 2002)) to the greatest extent of all TAS2R genes, it explains only $50-60 \%$ of individual ability to sensation the bitterness of $\mathrm{N}-\mathrm{C}=\mathrm{S}$ moiety (Knaapila et al., 2012). However, other bitter compounds without N$-\mathrm{C}=\mathrm{S}$ moiety, which may act as ligands for other bitter receptors, have also been described (Meyerhof et al., 2010). Furthermore, subjects who are highly sensitive to one bitter compound can be quite insensitive to another (Nolden et al., 2016). Various bitter taste receptors are involved in bitter sensation and the remaining variance in the ability to perceive bitterness, which is unexplained by the $\mathrm{N}-\mathrm{C}=\mathrm{S}$ bitterness perception, could be explained through the genetic variation of genes encoding other bitter taste receptors.

It has been shown that four SNPs, in TAS2R3 (rs765007), TAS2R4 (rs2234001), and in TAS2R5 (rs2234012 and rs2227264), which are localized in genomic locus $7 \mathrm{q} 34$, are in linkage disequilibrium and form a haploblock, which is independent of the TAS2R38 haploblock (Hayes et al., 2011). Although $T A S 2 R 3, T A S 2 R 4$, and TAS2R 5 remain orphan receptors, their engagement in the variability in bitterness perception of caffeine, capsaicin and ethanol has been demonstrated. However, the results are inconsistent (Hayes et al., 2011; 2015; Nolden et al., 2016). The SNPs, which are analyzed in the present study - the rs $765007(\mathrm{C} / \mathrm{T})$ in the TAS2R3 gene and the rs $2234012(\mathrm{~A} / \mathrm{G})$ in the TAS2R 5 gene, are located in the 5'UTR, a region that typically contains sequences that regulate translation efficiency or messenger RNA stability.

The other SNPs analyzed in the present study occur in the coding sequences of TAS2R19 (rs10772420) - (Hayes et al., 2015) and TAS2R50 (rs1376251) - (Schembre et al., 2013). The rs 10772420 of TAS2R19 is an A/G substitution, which causes an Arg to Cys substitution at a position 299 of the polypeptide. Individuals who are AA homozygotes rate grapefruit juice as twice as bitter as GG homozygotes (Hayes et al., 2015). The rs 1376251 of TAS2R 50 is a C/T substitution, which exchanges Cys for Tyr at a position 203 of the protein. The $\mathrm{C}$ allele is associated with decreased consumption of fruits or vegetables (Schembre et al., 2013) and is associated with a higher risk of cardiovascular disease development (Shiffman et al., 2005).

Our previous study showed an association between the frequency of intake of different types of bittertasting food items and polymorphisms of the TAS2R 38 and $C A 6$ genes in elderly women (Mikołajczyk-Stecyna et al., 2017). However, these associations did not explain all interindividual variance in bitter food intake. For this reason, we selected additional genes (TAS2R3, TAS2R5, TAS2R19 and TAS2R50) that might contribute to variability in bitter-tasting food intake (Hayes et al., 2011; Nolden et al., 2016; Ong et al., 2018 Schembre et al., 2013) and tested the association of selected polymorphisms (rs765007, rs2234012, rs10772420, rs 1376251) and the frequency of intake of bitter-tasting food in elderly women.

A connection between the consumption of bitter food such as Brassica vegetables, coffee and grapefruit with health outcomes has been already demonstrated (Alam et al., 2014; Choi et al., 2012; Dinkova-Kostova, 2013) and low consumption of bitter food might contribute to a higher prevalence of obesity and metabolic syndrome. Moreover, women are more sensitive to bitterness than men and may avoid bitter-tasting food more often than men (Barragán et al., 2018). Furthermore, the group selected for the present study are postmenopausal women. Menopause increases adiposity in women and thus changes lipid and glucose metabolism (Toth et al., 2000). Increased body fat is associated with a higher level of serum C-reactive protein (CRP), 
which is a marker of low level chronic inflammation (Yeh et al., 2003). For these reasons, elderly women are at an increased risk of cardiovascular diseases and both proper nutrition and weight management are particular challenges to elderly women's health.

To date, studies focusing on the effect of TAS2R genes polymorphisms on food choices in the elderly are limited. For these reasons, for the first time, we tested whether polymorphism of TAS2R3,TAS2R5, $T A S 2 R 19$ and TAS2R50 genes may be associated with variation in the intake of bitter-tasting food items among elderly women and whether polymorphism of selected TAS2R genes and bitter foods intake may influence the lipid profile, glucose and CRP concentration in blood.

\section{METHODS}

\section{Subjects and study design}

The research protocol was reviewed and approved by the Local Ethics Committee of Poznan University of Medical Sciences (approval no. 560/09). This study included 116 white female volunteers older than 60 years (mean \pm SD of age was $68.2 \pm 7.13$ ), who were enrolled at the University of the Third Age in Poznań. 60 years of age was chosen as the cut-off age, because it is the retirement age for women in Poland. Retirement may affect eating behavior and food choices and lead to a lower physical activity level, and together these result in weight gain (Chung et al., 2009) and an increase in body fat, especially in postmenopausal women (Toth et al., 2000). The group was enrolled for a different study performed previously (Chmurzynska et al., 2013). For this reason, the main exclusion criteria were treatments interfering with folate metabolism, megaloblastic anemia, regular intake of folic acid supplements, memory impairment and diabetes. Informed written consents, a general one and for genetic testing, were obtained from all participants and the data were analyzed anonymously.

Due to the sensitive nature of the questions asked in this study, survey respondents were assured that the raw data would remain confidential and would not be shared.

Body mass and height were measured using an electronic scale with a stadiometer (TP-150/1, Fawag, Poland). The subjects were fasting and dressed in light clothing during the measurements, which were recorded to the nearest $0.5 \mathrm{~cm}$ and $0.5 \mathrm{~kg}$. The body weight, height, body mass index (calculated as body weight in kilograms divided by height in meters squared, in accordance with the Clinical Guidelines on the Identification, Evaluation, and Treatment of Overweight and Obesity in Adults, 1998), were assessed in all participants.

\section{Bitter food and beverage intake}

The medians and interquartile ranges of BMI and blood biomarkers have been published elsewhere (Mikołajczyk-Stecyna et al., 2017). In brief, the median ( \pm interquartile range) BMI $\left(26.2 \mathrm{~kg} / \mathrm{m}^{2} \pm 4.93\right)$ was defined as overweight (according to World Health Organization, WHO). The median ( \pm interquartile range) of the serum concentration of total cholesterol and low density lipoprotein (LDL) cholesterol were borderline high $(239.0 \mathrm{mg} / \mathrm{dL} \pm 56.00$ and $143.0 \mathrm{mg} / \mathrm{dL}$ \pm 49.00 , respectively), but the values of high density lipoprotein (HDL) $(69.0 \mathrm{mg} / \mathrm{dL} \pm 23.00)$, triglycerides (TG) $(108.0 \mathrm{mg} / \mathrm{dL} \pm 61.00)$, and fasting glucose $(82.0$ $\mathrm{mg} / \mathrm{dL} \pm 14.00)$ fell in the optimal range, according to NCEP-III (Third Report of the National Cholesterol Education Program, 2002). The median ( \pm interquartile range) of serum C-reactive protein (CRP) level (4.0 $\mathrm{mg} / \mathrm{dL} \pm 0.80$ ) was above the optimal value (Yeh and Willerson, 2003).

The frequency of bitter food item intake was assessed using a food frequency questionnaire (FFQ) designed originally to assess folate intake. However, the list of products contained of the most popular bitter-tasting food products in Poland, like Brassica vegetables: white cabbage, Brussels sprouts, broccoli, and cauliflower, as well as grapefruit and coffee. A detailed description of the data collected from the FFQ has been presented elsewhere (Chmurzynska et al., 2013). The FFQ data were collected as a result of interviews with the participants conducted by trained dietitians. The median values and the interquartile ranges of the intake frequencies of these bitter foods have also been published previously (Mikołajczyk-Stecyna et al., 2017). In brief, the values of median frequency of eating bitter-tasting items counting as times per 30 days \pm interquartile range were: $10.0 \pm 9.0$ for Brassica vegetables in general, $30.0 \pm 0.0$ for coffee and $0.5 \pm 4.0$ for grapefruit. 


\section{Blood markers}

Overnight fasting blood samples were collected for biochemical parameter assessment. Serum glucose, total cholesterol, HDL cholesterol, LDL cholesterol, TG, and CRP concentrations were measured using a Vitalab Flexor biochemical analyzer (Vital Scientific, Spankeren, Netherlands).

\section{Genotyping}

DNA was isolated from blood lymphocytes using a $0.3-\mathrm{mL}$ Genomic Mini AX Blood Spin kit (A\&A Biotechnology, Gdynia, Poland). Four SNPs within the TAS2R3 gene (rs765007), the TAS2R5 gene (rs2234012), the TAS2R19 gene (rs10772420), and the TAS2R50 gene (rs1376251) were genotyped using the high resolution melting (HRM) method (LightCycler 480, Roche) with the sequencing of selected samples (Genomed, Poland) as positive controls. Primers were designed on the basis of the human gene sequences for the TAS2R3 (Gene ID: 50831) and TAS2R5 (Gene ID: 54429) genes (GeneBank accession number NC_000007.14), as well as for the TAS2R19 (ID: 259294) and TAS2R50 (Gene ID: 259296) genes (GeneBank accession number NC_000012.12), using Primer-Blast software. The primers for the genotyped SNPs are collected in Table 1.

HRM reactions were performed on genomic DNA separately for each SNP. The reaction mixtures contained 5 pmol of each primer, LightCycler 480 high resolution melting master (Roche; a ready-to-use $2 \times$ concentrated hot-start reaction mix), and varying concentrations of $\mathrm{Mg}^{2+}$ depending on the SNP $(2 \mathrm{mmol}$ for rs 765007 and rs $1376251 ; 2.5 \mathrm{mmol}$ for rs 2234012 and rs10772420) to optimize the reaction conditions. The reaction profile for all SNPs was preincubation at $95^{\circ} \mathrm{C}$ for $1 \mathrm{~min}$, followed by 45 cycles at $95^{\circ} \mathrm{C}$ for $10 \mathrm{~s}$, $64^{\circ} \mathrm{C}$ for $15 \mathrm{~s}, 72^{\circ} \mathrm{C}$ for $15 \mathrm{~s}$, and high resolution melting in the melting range of $70-90^{\circ} \mathrm{C}$ with a ramp rate of $0.02^{\circ} \mathrm{C} / \mathrm{s}$ and 25 acquisitions per ${ }^{\circ} \mathrm{C}$. The amplified DNA fragments were $117 \mathrm{bp}$ for rs765007 (TAS2R3), $129 \mathrm{bp}$ for rs 2234012 (TAS2R5), $182 \mathrm{bp}$ for rs 10772420 (TAS2R19), and 135 bp for rs1376251 (TAS2R50).

\section{Statistical analysis}

The distribution of intake frequencies, as well as the blood biomarkers and BMI, were checked for normality using the Shapiro-Wilk test with $\alpha=0.05$. Since the assumption of a normal distribution does not hold true for these data, and not all the groups that were studied contained more than 30 subjects, nonparametric tests were used (McClave and Sincich, 2003). The median and interquartile ranges are common summary measures for an outcome lacking normality, and so the data in this paper are presented as medians \pm interquartile range values (McClave and Sincich, 2003).

Only the dominant model of inheritance was considered in association testing, because it requires the smallest sample size to achieve $80 \%$ power compared to other genetic models (Hong and Park, 2012). The only exception was association analysis for TAS2R3. According to the SNP NCBI database, the $\mathrm{C}$ and $\mathrm{T}$ allele frequencies of rs765007 genetic polymorphism within $T A S 2 R 3$ are similar; the recessive model of inheritance was thus also analyzed. Because it has been shown that the SNPs studied within the TAS2R3 and TAS2R5 form a haploblock (Hayes et al., 2011) and SNPs within TAS2R19 and TAS2R50 gene are localized in the same locus, the combined effect of these two pairs of SNPs on the frequency of bitter food intake was also tested using a nonparametric Mann-Whitney test.

These analyses were performed for the study group as a whole, followed by analyses of the subgroups

Table 1. Primers for the single genetic polymorphism of selected TAS2R genotyping

\begin{tabular}{lll}
\hline \multicolumn{1}{c}{ SNP } & \multicolumn{1}{c}{ Primer forward } & \multicolumn{1}{c}{ Primer reverse } \\
\hline rs765007 (TAS2R3) & 5'GTGAAGCAACAGGTAGAGGAGT3' & 5'GGCAGCCCTGATCTTTGTTC3' \\
rs2234012 (TAS2R5) & 5'CTCTCTGTTCCTCATCACAGC3' & 5'TGAGGTCAGATCCCCTGGTA3' \\
rs10772420 (TAS2R19) & 5'TCTGCTAGAAGACCCACGATG3 & 5'CTGCTTTGCCAAACTGTTG3' \\
rs1376251 (TAS2R50) & 5'TGGTGCTGAGATCTTGCGAT3' & 5'TGACTGTAACTACCCTATGGAGC3' \\
\hline
\end{tabular}


created by stratifying the group by means of the median intake of Brassica vegetables in general, grapefruit or coffee. These three food groups were chosen, because they contain different bitter chemical compounds present: indole-3-carbinol in Brassica vegetables (Choi et al., 2012), naringin in grapefruit (Alam et al., 2014), and chlorogenic acid and caffeine in coffee (Kraehenbuehl et al., 2017), which can act as different ligands for bitter taste receptors. Subjects whose frequency of intake was equal to or greater than the median were assigned to the "above the median" group; the rest were assigned to the "below the median" group.

Due to the lack of a universal appropriate adjustment for multiplicity testing larger than 5 , and the great risk of reducing the power to detect existing effects caused by this adjustment (McClave and Sincich, 2003; Vasilopoulos et al., 2016), $P$ values $<0.05$ were taken as significant. Moreover, associations between different $T A S 2 R$ SNPs and food frequency intake or biomarkers were considered as separate hypotheses to eliminate the need for a severe multiple comparison correction to reduce Type I error. All analyses were performed using Statistica 13 software (Statsoft, Poland).

The present work was performed according to STROBE.

\section{RESULTS}

\section{Allele and genotype distributions}

The genotype distribution and allele frequencies of the SNPs of TAS2R3 (rs765007), TAS2R5 (rs2234012),
TAS2R19 (rs10772420) and TAS2R50 (rs1376251) are presented in Table 2. The allele frequencies were consistent with the data published in the SNP database (https://www.ncbi.nlm.nih.gov/snp/) for each of the SNPs tested.

\section{Associations between TAS2R3, TAS2R5, TAS2R19, and TAS2R50 genetic polymorphisms and intake of bitter-tasting foods}

The most frequently chosen bitter product among all genotype groups was coffee, and grapefruit was the least often chosen. Correspondences between monthly intake frequencies of bitter-tasting foods - Brassica vegetables in general, grapefruit, and coffee (shown as median \pm interquartile range) - and the genetic polymorphisms, are collected in Table 3.

Associations were observed between TAS2R3 genetic polymorphism and the frequency of Brassica vegetable intake and TAS2R5 genetic polymorphism and the frequency of grapefruit intake (Table 3 ). The $\mathrm{C}$ carriers of TAS2R 3 choose Brassica vegetables more frequently than the TT homozygotes did, while the G carriers of TAS $2 R 5$ chose grapefruit more frequently than the AA homozygotes. Association analysis of the effect of the combined TAS $2 R 3$ and TAS2R 5 genotypes on the intake of bitter products indicated that the $\mathrm{C}$ carriers of TAS2R 3 and the $\mathrm{G}$ carriers of TAS2R 5 ate Brassica vegetables more frequently than the respective TT and AA homozygotes. There were no associations between the genetic polymorphisms of TAS $2 R 19$ or TAS2R50 and frequency of bitter product intake.

Table 2. Genotype and allele distributions of TAS2R3, TAS2R5, TAS2R19, and TAS2R50 genes in the study group

\begin{tabular}{|c|c|c|c|c|c|}
\hline \multirow{2}{*}{$\begin{array}{r}\text { Gene } \\
T A S 2 R 3\end{array}$} & \multicolumn{3}{|c|}{ Genotype distribution $n, \%$} & \multicolumn{2}{|c|}{ Allele frequency } \\
\hline & $\mathrm{CC}$ & $\mathrm{CT}$ & $\mathrm{TT}$ & $\mathrm{C}$ & $\mathrm{T}$ \\
\hline rs765007 & $34(29.3)$ & $53(45.7)$ & $29(25)$ & 0.522 & 0.478 \\
\hline$T A S 2 R 5$ & AA & $\mathrm{AG}$ & GG & A & G \\
\hline rs 2234012 & $30(25.9)$ & $65(56.0)$ & $21(18.1)$ & 0.539 & 0.461 \\
\hline$T A S 2 R 19$ & $\mathrm{AA}$ & AG & GG & A & G \\
\hline rs 10772420 & 37 (31.9) & $62(53.5)$ & $17(14.6)$ & 0.586 & 0.414 \\
\hline$T A S 2 R 50$ & $\mathrm{CC}$ & $\mathrm{CT}$ & $\mathrm{TT}$ & $\mathrm{C}$ & $\mathrm{T}$ \\
\hline rs1376251 & $48(41.4)$ & $50(43.1)$ & $18(15.5)$ & 0.629 & 0.371 \\
\hline
\end{tabular}


Table 3. Associations between TAS2R3, TAS2R5, TAS2R19, TAS2R50 genetic polymorphisms and the monthly frequency of Brassica sp., grapefruit and coffee intake

\begin{tabular}{|c|c|c|c|c|c|c|c|}
\hline Genotype/Diplotype & $n$ & $\begin{array}{c}\text { Brassica } \text { sp. } \\
\text { in general frequency } \\
\text { per month }\end{array}$ & $P$ & $\begin{array}{l}\text { Grapefruit } \\
\text { frequency } \\
\text { per month }\end{array}$ & $P$ & $\begin{array}{c}\text { Coffee } \\
\text { frequency } \\
\text { per month }\end{array}$ & $P$ \\
\hline \multicolumn{8}{|l|}{$T A S 2 R 3$} \\
\hline $\mathrm{CC}+\mathrm{CT}$ & 87 & $10.0 \pm 10.0$ & 0.038 & $1.0 \pm 4.0$ & 0.732 & $30.0 \pm 14.0$ & 0.859 \\
\hline $\mathrm{TT}$ & 29 & $6.0 \pm 8.0$ & & $0 \pm 4.0$ & & $30.0 \pm 10.0$ & \\
\hline $\mathrm{CC}$ & 34 & $10.5 \pm 10.0$ & & $0 \pm 4.0$ & & $30.0 \pm 14.0$ & $\mathrm{~s}$ \\
\hline $\mathrm{CT}+\mathrm{TT}$ & 82 & $9.5 \pm 7.0$ & 0.259 & $1.0 \pm 4.0$ & 0.969 & $30.0 \pm 14.0$ & 0.997 \\
\hline \multicolumn{8}{|l|}{ TAS2R5 } \\
\hline $\mathrm{GG}+\mathrm{AG}$ & 86 & $10.0 \pm 10.0$ & 0.138 & $1.0 \pm 4.0$ & 0.048 & $30.0 \pm 14.0$ & 0.640 \\
\hline AA & 30 & $9.0 \pm 8.0$ & & $0 \pm 1.0$ & & $30.0 \pm 0$ & \\
\hline \multicolumn{8}{|l|}{ TAS2R19 } \\
\hline $\mathrm{GG}+\mathrm{AG}$ & 79 & $9.0 \pm 9.0$ & 0.066 & $1.0 \pm 4.0$ & 0.595 & $30.0 \pm 14.0$ & 0.939 \\
\hline AA & 37 & $11.0 \pm 8.0$ & & $0 \pm 2.0$ & & $30.0 \pm 0$ & \\
\hline \multicolumn{8}{|l|}{ TAS2R50 } \\
\hline $\mathrm{TT}+\mathrm{CT}$ & 68 & $10.0 \pm 10.0$ & 0.865 & $1.0 \pm 4.0$ & 0.613 & $30.0 \pm 10.0$ & 0.617 \\
\hline $\mathrm{CC}$ & 48 & $9.5 \pm 8.0$ & & $0.5 \pm 2.0$ & & $30.0 \pm 20.0$ & \\
\hline \multicolumn{8}{|l|}{$T A S 2 R 3 \& T A S 2 R 5$} \\
\hline (3) $\mathrm{CC}+\mathrm{CT} \&$ (5) $\mathrm{GG}+\mathrm{AG}$ & 66 & $10.0 \pm 7.0$ & 0.008 & $1.0 \pm 4.0$ & 0.674 & $30.0 \pm 14.0$ & 0.866 \\
\hline (3) $\mathrm{TT} \&$ (5) AA & 9 & $6.0 \pm 3.0$ & & $1.0 \pm 4.0$ & & $30.0 \pm 14.0$ & \\
\hline \multicolumn{8}{|l|}{$T A S 2 R 19 \& \operatorname{TAS} 2 R 50$} \\
\hline (19) $\mathrm{GG}+\mathrm{AG} \&$ (50) $\mathrm{TT}+\mathrm{CT}$ & 61 & $10.0 \pm 9.0$ & 0.387 & $1.0 \pm 4.0$ & 0.473 & $30.0 \pm 10.0$ & 0.692 \\
\hline (19) AA \& (50) CC & 30 & $11.0 \pm 11.0$ & & $0 \pm 2.0$ & & $30.0 \pm 14.0$ & \\
\hline
\end{tabular}

Statistically significant results are in bold.

Associations between TAS2R3, TAS2R5, TAS2R19, and TAS2R 50 polymorphisms, intake of bitter-tasting foods, and blood biochemical biomarkers

The relationships between genetic polymorphisms, bitter foods intake, and biochemical biomarkers in blood are shown in Table 4. The TT homozygotes of the TAS2R3 gene have higher CRP levels than the $\mathrm{C}$ carriers. This association was only observed in groups with a lower frequency intake of all the bitter-tasting products being examined. Of those subjects who chose coffee more frequently, the TT homozygotes had higher total cholesterol and LDL cholesterol concentrations in serum than the $\mathrm{C}$ carriers (Table 4).

Those subjects who chose Brassica vegetables or grapefruit less frequently and were $G$ carriers of the TAS2R 5 genetic polymorphism had higher serum levels of CRP than the AA homozygotes (Table 4).

The association between TAS2R19 gene polymorphism and the glucose serum level was only noted for subjects who ate grapefruit with a frequency above the median value. The $\mathrm{G}$ carriers had lower glucose levels in serum than the AA homozygotes (Table 4). 
Mikołajczyk-Stecyna, J., Malinowska, A. M., Chmurzynska, A. (2020). Polymorphism of TAS2R3, TAS2R5, TAS2R19, and TAS2R50 genes and bitter food intake frequency in elderly woman. Acta Sci. Pol. Technol. Aliment., 19(1), 109-122. http://dx.doi. org/10.17306/J.AFS.2020.0729

Table 4. Associations between $T A S 2 R 3(\mathrm{C} / \mathrm{T}), \operatorname{TAS} 2 R 5(\mathrm{~A} / \mathrm{G}), \operatorname{TAS} 2 R 50(\mathrm{C} / \mathrm{T})$, and $T A S 2 R 19(\mathrm{~A} / \mathrm{G})$ polymorphisms and blood biomarkers by median monthly intake frequency of bitter products; statistically significant results are in bold

\begin{tabular}{|c|c|c|c|c|c|c|c|c|c|c|}
\hline \multicolumn{11}{|c|}{$T A S 2 R 3$} \\
\hline \multirow{2}{*}{ Marker } & \multicolumn{5}{|c|}{ Brassica sp. monthly intake frequency $<$ median (10) } & \multicolumn{5}{|c|}{ Brassica sp. monthly intake frequency $\geq$ median (10) } \\
\hline & $n$ & $\mathrm{CC}+\mathrm{CT}$ & $n$ & TT & $P$ & $n$ & $\mathrm{CC}+\mathrm{CT}$ & $n$ & TT & $P$ \\
\hline T Chol, mg/dL & \multirow{6}{*}{37} & $254.0 \pm 40.0$ & \multirow{6}{*}{18} & $231.0 \pm 44.0$ & 0.175 & \multirow{6}{*}{50} & $230.0 \pm 64.0$ & \multirow{6}{*}{11} & $253.0 \pm 76.0$ & 0.606 \\
\hline $\mathrm{HDL}, \mathrm{mg} / \mathrm{dL}$ & & $65.0 \pm 18.0$ & & $67.0 \pm 27.0$ & 0.279 & & $71.5 \pm 22.0$ & & $69.0 \pm 19.0$ & 0.619 \\
\hline $\mathrm{LDL}, \mathrm{mg} / \mathrm{dL}$ & & $136.0 \pm 36.0$ & & $157.0 \pm 33.0$ & 0.043 & & $137.0 \pm 52.00$ & & $168.0 \pm 79.0$ & 0.093 \\
\hline $\mathrm{TG}, \mathrm{mg} / \mathrm{dL}$ & & $135.0 \pm 90.0$ & & $125.0 \pm 59.0$ & 0.542 & & $91.5 \pm 61.0$ & & $120.0 \pm 55.0$ & 0.059 \\
\hline Glucose, $\mathrm{mg} / \mathrm{dL}$ & & $84 \pm 18.0$ & & $83.5 \pm 10.0$ & 0.699 & & $81.5 \pm 14.0$ & & $84.0 \pm 17.0$ & 0.272 \\
\hline $\mathrm{CRP}, \mathrm{mg} / \mathrm{L}$ & & $3.9 \pm 0.5$ & & $4.3 \pm 0.5$ & 0.044 & & $3.9 \pm 0.8$ & & $4.0 \pm 0.7$ & 0.218 \\
\hline \multirow{2}{*}{ Marker } & \multicolumn{5}{|c|}{ Coffee monthly intake frequency $<$ median $(30)$} & \multicolumn{5}{|c|}{ Coffee monthly intake frequency $\geq$ median (30) } \\
\hline & $n$ & $\mathrm{CC}+\mathrm{CT}$ & $n$ & TT & $P$ & $n$ & $\mathrm{CC}+\mathrm{CT}$ & $n$ & TT & $P$ \\
\hline T Chol, mg/dL & \multirow{6}{*}{24} & $249.0 \pm 53.5$ & \multirow{6}{*}{8} & $243.5 \pm 75.0$ & 0.982 & \multirow{6}{*}{63} & $239.0 \pm 63.0$ & \multirow{6}{*}{21} & $262.0 \pm 48.00$ & 0.036 \\
\hline $\mathrm{HDL}, \mathrm{mg} / \mathrm{dL}$ & & $65.0 \pm 19.0$ & & $63.0 \pm 25.5$ & 0.556 & & $69.0 \pm 24.0$ & & $69.0 \pm 23.0$ & 0.638 \\
\hline $\mathrm{LDL}, \mathrm{mg} / \mathrm{dL}$ & & $145.0 \pm 38.0$ & & $143.0 \pm 56.0$ & 0.586 & & $147.0 \pm 48.0$ & & $173.0 \pm 59.0$ & 0.026 \\
\hline $\mathrm{TG}, \mathrm{mg} / \mathrm{dL}$ & & $118.0 \pm 115.0$ & & $110.0 \pm 39.0$ & 0.983 & & $106.0 \pm 55.0$ & & $125.0 \pm 65.0$ & 0.679 \\
\hline Glucose, $\mathrm{mg} / \mathrm{dL}$ & & $85.0 \pm 15.0$ & & $85.0 \pm 16.0$ & 0.931 & & $81.0 \pm 12.0$ & & $84.0 \pm 12.0$ & 0.377 \\
\hline $\mathrm{CRP}, \mathrm{mg} / \mathrm{L}$ & & $4.0 \pm 0.5$ & & $4.4 \pm 0.5$ & 0.022 & & $3.9 \pm 0.9$ & & $4.1 \pm 0.5$ & 0.135 \\
\hline \multirow{2}{*}{ Marker } & \multicolumn{5}{|c|}{ Grapefruit monthly intake frequency $<$ median $(0.5)$} & \multicolumn{5}{|c|}{ Grapefruit monthly intake frequency $\geq$ median $(0.5)$} \\
\hline & $n$ & $\mathrm{CC}+\mathrm{CT}$ & $n$ & TT & $P$ & $n$ & $\mathrm{CC}+\mathrm{CT}$ & $n$ & TT & $P$ \\
\hline T Chol, mg/dL & \multirow{6}{*}{42} & $240.0 \pm 60.0$ & \multirow{6}{*}{15} & $253.0 \pm 62.0$ & 0.404 & \multirow{6}{*}{45} & $240.0 \pm 54.0$ & \multirow{6}{*}{14} & $230.0 \pm 44.0$ & 0.137 \\
\hline $\mathrm{HDL}, \mathrm{mg} / \mathrm{dL}$ & & $65.0 \pm 29.0$ & & $61.0 \pm 26.0$ & 0.703 & & $69.0 \pm 13.0$ & & $69.0 \pm 27.0$ & 0.649 \\
\hline $\mathrm{LDL}, \mathrm{mg} / \mathrm{dL}$ & & $144.5 \pm 51.0$ & & $163.0 \pm 58.0$ & 0.142 & & $153.0 \pm 40.0$ & & $130.5 \pm 36.0$ & 0.071 \\
\hline $\mathrm{TG}, \mathrm{mg} / \mathrm{dL}$ & & $118.0 \pm 86.0$ & & $122.0 \pm 38.0$ & 0.913 & & $97.0 \pm 66.0$ & & $118.5 \pm 59.0$ & 0.195 \\
\hline Glucose, $\mathrm{mg} / \mathrm{dL}$ & & $84.0 \pm 14.0$ & & $84.0 \pm 13.0$ & 0.697 & & $82.0 \pm 12.0$ & & $82.5 \pm 15.0$ & 0.656 \\
\hline $\mathrm{CRP}, \mathrm{mg} / \mathrm{L}$ & & $3.8 \pm 0.8$ & & $4.0 \pm 1.0$ & 0.007 & & $4.0 \pm 0.6$ & & $4.3 \pm 0.6$ & 0.081 \\
\hline \multicolumn{11}{|c|}{$T A S 2 R 5$} \\
\hline \multirow{2}{*}{ Marker } & \multicolumn{5}{|c|}{ Brassica sp. monthly intake frequency $<$ median $(10)$} & \multicolumn{5}{|c|}{ Brassica sp. monthly intake frequency $\geq$ median (10) } \\
\hline & $n$ & $\mathrm{GG}+\mathrm{AG}$ & $n$ & $\mathrm{AA}$ & $P$ & $n$ & $\mathrm{GG}+\mathrm{AG}$ & $\mathrm{n}$ & AA & $P$ \\
\hline T Chol, mg/dL & \multirow{6}{*}{39} & $246.0 \pm 53.0$ & & $244.0 \pm 44.5$ & 0.781 & & $229.0 \pm 65.0$ & & $245.0 \pm 49.0$ & 0.476 \\
\hline $\mathrm{HDL}, \mathrm{mg} / \mathrm{dL}$ & & $65.0 \pm 27.0$ & & $66.0 \pm 19.0$ & 0.393 & & $71.0 \pm 22.0$ & & $69.0 \pm 19.0$ & 0.844 \\
\hline $\mathrm{LDL}, \mathrm{mg} / \mathrm{dL}$ & & $143.0 \pm 43.0$ & 16 & $157.0 \pm 36.5$ & 0.623 & 17 & $136.0 \pm 57.0$ & & $149.5 \pm 48.0$ & 0.316 \\
\hline $\mathrm{TG}, \mathrm{mg} / \mathrm{dL}$ & & $128.0 \pm 78.0$ & & $136.0 \pm 73.0$ & 0.419 & 年 & $92.0 \pm 58.0$ & T & $102.0 \pm 75.0$ & 0.944 \\
\hline Glucose, $\mathrm{mg} / \mathrm{dL}$ & & $82.0 \pm 13.0$ & & $87.0 \pm 15.0$ & 0.200 & & $82.0 \pm 13.0$ & & $79.5 \pm 21.0$ & 0.795 \\
\hline $\mathrm{CRP}, \mathrm{mg} / \mathrm{L}$ & & $4.1 \pm 0.7$ & & $3.8 \pm 0.5$ & 0.006 & & $4.0 \pm 0.8$ & & $3.9 \pm 0.7$ & 0.463 \\
\hline
\end{tabular}


Mikołajczyk-Stecyna, J., Malinowska, A. M., Chmurzynska, A. (2020). Polymorphism of TAS2R3, TAS2R5, TAS2R19, and TAS2R50 genes and bitter food intake frequency in elderly woman. Acta Sci. Pol. Technol. Aliment., 19(1), 109-122. http://dx.doi. org/10.17306/J.AFS.2020.0729

Table 4 - cont.

\begin{tabular}{|c|c|c|c|c|c|c|c|c|c|c|}
\hline \multirow{2}{*}{ Marker } & \multicolumn{5}{|c|}{ Coffee monthly intake frequency $<$ median $(30)$} & \multicolumn{5}{|c|}{ Coffee monthly intake frequency $\geq$ median (30) } \\
\hline & $n$ & $\mathrm{GG}+\mathrm{AG}$ & $n$ & $\mathrm{AA}$ & $P$ & $n$ & $\mathrm{GG}+\mathrm{AG}$ & $n$ & AA & $P$ \\
\hline $\mathrm{T}$ Chol, mg/dL & \multirow{6}{*}{25} & $246.0 \pm 59.0$ & \multirow{6}{*}{7} & $252.0 \pm 41.0$ & 0.689 & \multirow{6}{*}{61} & $238.0 \pm 62.0$ & \multirow{6}{*}{23} & $241.0 \pm 46.0$ & 0.824 \\
\hline $\mathrm{HDL}, \mathrm{mg} / \mathrm{dL}$ & & $65.0 \pm 30.0$ & & $65.0 \pm 11.0$ & 0.989 & & $70.0 \pm 22.0$ & & $69.0 \pm 26.0$ & 0.757 \\
\hline $\mathrm{LDL}, \mathrm{mg} / \mathrm{dL}$ & & $143.0 \pm 40.0$ & & $140.0 \pm 39.0$ & 0.846 & & $139.0 \pm 54.0$ & & $159.0 \pm 47.0$ & 0.624 \\
\hline $\mathrm{TG}, \mathrm{mg} / \mathrm{dL}$ & & $108.0 \pm 82.0$ & & $136.0 \pm 78.0$ & 0.123 & & $108.0 \pm 58.0$ & & $108.0 \pm 58.0$ & 0.978 \\
\hline Glucose, $\mathrm{mg} / \mathrm{dL}$ & & $82.0 \pm 14.0$ & & $89.0 \pm 10.0$ & 0.542 & & $82.0 \pm 11.0$ & & $84.0 \pm 22.0$ & 0.963 \\
\hline $\mathrm{CRP}, \mathrm{mg} / \mathrm{L}$ & & $4.1 \pm 0.7$ & & $3.8 \pm 0.5$ & 0.006 & & $4.0 \pm 0.8$ & & $3.9 \pm 0.7$ & 0.075 \\
\hline \multirow{2}{*}{ Marker } & \multicolumn{5}{|c|}{ Grapefruit monthly intake frequency $<$ median $(0.5)$} & \multicolumn{5}{|c|}{ Grapefruit monthly intake frequency $\geq$ median $(0.5)$} \\
\hline & $n$ & $\mathrm{GG}+\mathrm{AG}$ & $n$ & AA & $P$ & $n$ & $\mathrm{GG}+\mathrm{AG}$ & $n$ & AA & $P$ \\
\hline T Chol, mg/dL & \multirow{6}{*}{38} & $248.0 \pm 65.0$ & \multirow{6}{*}{19} & $241.0 \pm 47.0$ & 0.762 & \multirow{6}{*}{48} & $233.5 \pm 59.0$ & \multirow{6}{*}{11} & $254.0 \pm 34.0$ & 0.357 \\
\hline $\mathrm{HDL}, \mathrm{mg} / \mathrm{dL}$ & & $65.0 \pm 28.0$ & & $69.0 \pm 25.0$ & 0.829 & & $70.0 \pm 19.5$ & & $66.0 \pm 9.0$ & 0.523 \\
\hline $\mathrm{LDL}, \mathrm{mg} / \mathrm{dL}$ & & $146.0 \pm 59.0$ & & $149.0 \pm 53.0$ & 0.697 & & $138.5 \pm 44.5$ & & $161.0 \pm 39.0$ & 0.213 \\
\hline $\mathrm{TG}, \mathrm{mg} / \mathrm{dL}$ & & $115.0 \pm 53.0$ & & $132.0 \pm 74.0$ & 0.542 & & $105.0 \pm 68.5$ & & $100.0 \pm 55.0$ & 0.673 \\
\hline Glucose, $\mathrm{mg} / \mathrm{dL}$ & & $82.5 \pm 12.0$ & & $87.0 \pm 21.0$ & 0.744 & & $81.5 \pm 12.0$ & & $84.0 \pm 24.0$ & 0.722 \\
\hline $\mathrm{CRP}, \mathrm{mg} / \mathrm{L}$ & & $4.0 \pm 1.00$ & & $3.8 \pm 0.6$ & 0.049 & & $4.1 \pm 0.6$ & & $4.0 \pm 0.8$ & 0.704 \\
\hline \multicolumn{11}{|c|}{ TAS2R19 } \\
\hline \multirow{2}{*}{ Marker } & \multicolumn{5}{|c|}{ Brassica sp. monthly intake frequency $<$ median $(10)$} & \multicolumn{5}{|c|}{ Brassica sp. monthly intake frequency $\geq$ median (10) } \\
\hline & $n$ & $\mathrm{GG}+\mathrm{AG}$ & $n$ & AA & $P$ & $n$ & $\mathrm{GG}+\mathrm{AG}$ & $n$ & $\mathrm{AA}$ & $P$ \\
\hline T Chol, mg/dL & \multirow{6}{*}{40} & $249.5 \pm 41.0$ & \multirow{6}{*}{14} & $232.0 \pm 54.0$ & 0.453 & \multirow{6}{*}{39} & $236.0 \pm 64.0$ & \multirow{6}{*}{23} & $231.0 \pm 70.0$ & 0.333 \\
\hline $\mathrm{HDL}, \mathrm{mg} / \mathrm{dL}$ & & $64.0 \pm 21.5$ & & $71.0 \pm 25.0$ & 0.258 & & $72.0 \pm 22.0$ & & $71.0 \pm 20.0$ & 0.752 \\
\hline $\mathrm{LDL}, \mathrm{mg} / \mathrm{dL}$ & & $155.0 \pm 40.0$ & & $145.0 \pm 40.0$ & 0.322 & & $138.5 \pm 48.0$ & & $135.0 \pm 69.0$ & 0.638 \\
\hline $\mathrm{TG}, \mathrm{mg} / \mathrm{dL}$ & & $131.5 \pm 89.0$ & & $124.0 \pm 101.0$ & 0.655 & & $91.5 \pm 57.0$ & & $112.0 \pm 64.0$ & 0.227 \\
\hline Glucose, $\mathrm{mg} / \mathrm{dL}$ & & $83.5 \pm 16.5$ & & $86.0 \pm 14.0$ & 0.748 & & $80.5 \pm 11.0$ & & $84.0 \pm 17.0$ & 0.345 \\
\hline $\mathrm{CRP}, \mathrm{mg} / \mathrm{L}$ & & $4.0 \pm 0.9$ & & $4.1 \pm 0.7$ & 0.824 & & $3.9 \pm 0.7$ & & $4.0 \pm 0.7$ & 0.652 \\
\hline \multirow{2}{*}{ Marker } & \multicolumn{5}{|c|}{ Coffee monthly intake frequency $<$ median $(30)$} & \multicolumn{5}{|c|}{ Coffee monthly intake frequency $\geq$ median $(30)$} \\
\hline & $n$ & $\mathrm{GG}+\mathrm{AG}$ & $n$ & AA & $P$ & $n$ & $\mathrm{GG}+\mathrm{AG}$ & $n$ & AA & $P$ \\
\hline $\mathrm{T}$ Chol, mg/dL & \multirow{6}{*}{23} & $252.0 \pm 54.0$ & \multirow{6}{*}{9} & $229.0 \pm 103.0$ & 0.245 & & $240.5 \pm 57.0$ & & $233.5 \pm 60.5$ & 0.448 \\
\hline $\mathrm{HDL}, \mathrm{mg} / \mathrm{dL}$ & & $63.0 \pm 21.0$ & & $74.0 \pm 16.0$ & 0.217 & & $69.0 \pm 23.5$ & & $70.0 \pm 22.0$ & 0.751 \\
\hline $\mathrm{LDL}, \mathrm{mg} / \mathrm{dL}$ & & $143.0 \pm 36.0$ & & $140.0 \pm 62.0$ & 0.525 & 56 & $146.5 \pm 46$ & & $141.0 \pm 59.0$ & 0.645 \\
\hline $\mathrm{TG}, \mathrm{mg} / \mathrm{dL}$ & & $133.0 \pm 97.0$ & & $105.0 \pm 52.0$ & 0.422 & 50 & $106.0 \pm 56.0$ & 28 & $121.0 \pm 64.0$ & 0.321 \\
\hline Glucose, $\mathrm{mg} / \mathrm{dL}$ & & $82.0 \pm 13.0$ & & $93.0 \pm 37.0$ & 0.624 & & $81.0 \pm 11.0$ & & $84.0 \pm 13.0$ & 0.543 \\
\hline $\mathrm{CRP}, \mathrm{mg} / \mathrm{L}$ & & $4.1 \pm 0.7$ & & $4.1 \pm 0.4$ & 0.785 & & $3.9 \pm 0.9$ & & $4.0 \pm 0.7$ & 0.736 \\
\hline
\end{tabular}


Mikołajczyk-Stecyna, J., Malinowska, A. M., Chmurzynska, A. (2020). Polymorphism of TAS2R3, TAS2R5, TAS2R19, and TAS2R50 genes and bitter food intake frequency in elderly woman. Acta Sci. Pol. Technol. Aliment., 19(1), 109-122. http://dx.doi. org/10.17306/J.AFS.2020.0729

Table 4 - cont.

\begin{tabular}{|c|c|c|c|c|c|c|c|c|c|c|}
\hline \multirow{2}{*}{ Marker } & \multicolumn{5}{|c|}{ Grapefruit monthly intake frequency $<$ median $(0.5)$} & \multicolumn{5}{|c|}{ Grapefruit monthly intake frequency $\geq$ median $(0.5)$} \\
\hline & $n$ & $\mathrm{GG}+\mathrm{AG}$ & $n$ & AA & $P$ & $n$ & $\mathrm{GG}+\mathrm{AG}$ & $n$ & AA & $P$ \\
\hline $\mathrm{T}$ Chol, mg/dL & \multirow{6}{*}{39} & $249.0 \pm 52.0$ & \multirow{6}{*}{19} & $228.0 \pm 62.0$ & 0.553 & \multirow{6}{*}{40} & $238.5 \pm 55.0$ & \multirow{6}{*}{18} & $237.0 \pm 62.0$ & 0.733 \\
\hline $\mathrm{HDL}, \mathrm{mg} / \mathrm{dL}$ & & $64.5 \pm 28.0$ & & $75.0 \pm 26.0$ & 0.323 & & $69.5 \pm 20.0$ & & $69.0 \pm 12.0$ & 0.821 \\
\hline $\mathrm{LDL}, \mathrm{mg} / \mathrm{dL}$ & & $153.5 \pm 53.0$ & & $135.0 \pm 58.0$ & 0.158 & & $138.5 \pm 41.0$ & & $148.5 \pm 67.0$ & 0.634 \\
\hline $\mathrm{TG}, \mathrm{mg} / \mathrm{dL}$ & & $129.5 \pm 64.0$ & & $120.0 \pm 67.0$ & 0.345 & & $92.0 \pm 60.0$ & & $113.5 \pm 70.0$ & 0.215 \\
\hline Glucose, $\mathrm{mg} / \mathrm{dL}$ & & $84.0 \pm 12.0$ & & $84.0 \pm 17.0$ & 0.361 & & $79.5 \pm 10.0$ & & $86.5 \pm 16.0$ & 0.023 \\
\hline $\mathrm{CRP}, \mathrm{mg} / \mathrm{L}$ & & $3.9 \pm 1.0$ & & $3.9 \pm 0.6$ & 0.748 & & $4.1 \pm 0.6$ & & $4.1 \pm 0.5$ & 0.645 \\
\hline \multicolumn{11}{|c|}{ TAS2R50 } \\
\hline \multirow{2}{*}{ Marker } & \multicolumn{5}{|c|}{ Brassica sp. monthly intake frequency $<$ median (10) } & \multicolumn{5}{|c|}{ Brassica sp. monthly intake frequency $\geq$ median (10) } \\
\hline & $n$ & $\mathrm{TT}+\mathrm{CT}$ & $n$ & $\mathrm{CC}$ & $P$ & $n$ & $\mathrm{TT}+\mathrm{CT}$ & $n$ & $\mathrm{CC}$ & $P$ \\
\hline $\mathrm{T}$ Chol, mg/dL & \multirow{6}{*}{29} & $254.0 \pm 49.0$ & \multirow{6}{*}{25} & $240.0 \pm 41.0$ & 0.635 & \multirow{6}{*}{39} & $245.5 \pm 63.0$ & \multirow{6}{*}{23} & $231.0 \pm 53.0$ & 0.344 \\
\hline $\mathrm{HDL}, \mathrm{mg} / \mathrm{dL}$ & & $63.0 \pm 23.0$ & & $68.0 \pm 24.0$ & 0.452 & & $70.5 \pm 14.0$ & & $76.0 \pm 27.0$ & 0.269 \\
\hline $\mathrm{LDL}, \mathrm{mg} / \mathrm{dL}$ & & $157.0 \pm 45.0$ & & $140.0 \pm 39.0$ & 0.603 & & $141.0 \pm 53.0$ & & $137.0 \pm 60.0$ & 0.320 \\
\hline $\mathrm{TG}, \mathrm{mg} / \mathrm{dL}$ & & $135.0 \pm 82.0$ & & $108.0 \pm 56.0$ & 0.542 & & $107.0 \pm 49.0$ & & $76.0 \pm 73.0$ & 0.032 \\
\hline Glucose, $\mathrm{mg} / \mathrm{dL}$ & & $84.0 \pm 18.0$ & & $84.0 \pm 13.0$ & 0.536 & & $82.0 \pm 12.0$ & & $81.0 \pm 15.0$ & 0.759 \\
\hline $\mathrm{CRP}, \mathrm{mg} / \mathrm{L}$ & & $4.0 \pm 0.8$ & & $4.0 \pm 0.6$ & 0.724 & & $4.0 \pm 0.6$ & & $4.1 \pm 0.8$ & 0.564 \\
\hline \multirow{2}{*}{ Marker } & \multicolumn{5}{|c|}{ Coffee monthly intake frequency $<$ median (30) } & \multicolumn{5}{|c|}{ Coffee monthly intake frequency $\geq$ median (30) } \\
\hline & $n$ & $\mathrm{TT}+\mathrm{CT}$ & $n$ & $\mathrm{CC}$ & $P$ & $n$ & $\mathrm{TT}+\mathrm{CT}$ & $n$ & $\mathrm{CC}$ & $P$ \\
\hline $\mathrm{T}$ Chol, mg/dL & \multirow{6}{*}{20} & $252.0 \pm 59.0$ & & $246.0 \pm 54.0$ & 0.334 & & $252.0 \pm 57.5$ & & $236.0 \pm 60.0$ & 0.522 \\
\hline $\mathrm{HDL}, \mathrm{mg} / \mathrm{dL}$ & & $63.0 \pm 20.0$ & & $75.0 \pm 35.0$ & 0.084 & & $70.0 \pm 23.5$ & & $69.0 \pm 22.0$ & 0.458 \\
\hline $\mathrm{LDL}, \mathrm{mg} / \mathrm{dL}$ & & $143.0 \pm 50.0$ & & $143.0 \pm 29.0$ & 0.648 & 48 & $156.0 \pm 52.0$ & & $137.0 \pm 52.0$ & 0.211 \\
\hline $\mathrm{TG}, \mathrm{mg} / \mathrm{dL}$ & & $134.0 \pm 82.00$ & 13 & $86.0 \pm 41.0$ & 0.012 & 48 & $109.0 \pm 57.0$ & J & $100.0 \pm 70.0$ & 0.315 \\
\hline Glucose, $\mathrm{mg} / \mathrm{dL}$ & & $84.0 \pm 25.0$ & & $86.0 \pm 13.0$ & 0.751 & & $88.5 \pm 12.0$ & & $82.0 \pm 15.0$ & 0.199 \\
\hline $\mathrm{CRP}, \mathrm{mg} / \mathrm{L}$ & & $4.1 \pm 0.7$ & & $4.2 \pm 0.7$ & 0.556 & & $4.0 \pm 0.8$ & & $4.0 \pm 1.0$ & 0.586 \\
\hline \multirow{2}{*}{ Marker } & \multicolumn{5}{|c|}{ Grapefruit monthly intake frequency $<$ median $(0.5)$} & \multicolumn{5}{|c|}{ Grapefruit monthly intake frequency $\geq$ median $(0.5)$} \\
\hline & $n$ & $\mathrm{TT}+\mathrm{CT}$ & $n$ & $\mathrm{CC}$ & $P$ & 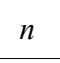 & $\mathrm{TT}+\mathrm{CT}$ & $n$ & $\mathrm{CC}$ & $P$ \\
\hline T Chol, mg/dL & \multirow{6}{*}{33} & $257.0 \pm 48.0$ & & $224.0 \pm 53.0$ & 0.002 & \multirow{6}{*}{35} & $229.5 \pm 59.0$ & \multirow{6}{*}{25} & $240.0 \pm 37.0$ & 0.513 \\
\hline $\mathrm{HDL}, \mathrm{mg} / \mathrm{dL}$ & & $65.0 \pm 22.0$ & & $76.0 \pm 31.0$ & 0.423 & & $70.0 \pm 15.0$ & & $69.0 \pm 17.0$ & 0.265 \\
\hline $\mathrm{LDL}, \mathrm{mg} / \mathrm{dL}$ & & $161.0 \pm 41.0$ & 23 & $124.0 \pm 51.0$ & 0.014 & & $135.0 \pm 48.0$ & & $150.0 \pm 30.0$ & 0.158 \\
\hline $\mathrm{TG}, \mathrm{mg} / \mathrm{dL}$ & & $131.0 \pm 74.0$ & & $107.0 \pm 70.0$ & 0.037 & & $107.5 \pm 53.0$ & & $93.0 \pm 72.0$ & 0.256 \\
\hline Glucose, $\mathrm{mg} / \mathrm{dL}$ & & $84.0 \pm 14.0$ & & $84.0 \pm 16.0$ & 0.722 & & $81.0 \pm 12.0$ & & $82.0 \pm 15.0$ & 0.687 \\
\hline CRP, mg/L & & $3.9 \pm 0.7$ & & $3.9 \pm 1$ & 0.678 & & $4.1 \pm 0.8$ & & $4.1 \pm 0.6$ & 0.425 \\
\hline
\end{tabular}


T carriers of TAS2R50 had a less favorable serum lipid profile, in that they had higher total cholesterol, LDL cholesterol, and TG levels than the CC homozygotes. These associations were observed in subjects with higher levels of intake of Brassica vegetables and with a lower intake of coffee and grapefruit.

\section{DISCUSSION}

Here we have investigated the associations between genetic polymorphisms located in the genes of bitter taste receptors (TAS2R3, TAS2R5, TAS2R19, and TAS2R50), intake of Brassica vegetables, grapefruit and coffee, and selected metabolic biomarkers in elderly women. Our paper is the first to investigate such associations in elderly people. Moreover, the associations between genetic polymorphisms within TAS2R3, TAS2R5, TAS2R19 and TAS2R50 genes and Brassica vegetable intake have not been tested previously. All the selected food products are bitter, but contain different bitter chemical compounds: indole-3-carbinol in Brassica vegetables (Choi et al., 2012), naringin in grapefruit, (Alam et al., 2014), chlorogenic acid and caffeine (Kraehenbuehl et al., 2017) and may contribute to different metabolic pathways. Moreover, selected food items have divergent flavors and dissimilar positive or negative social and cultural associations (Rabin et al., 2007). The food items selected for analysis in our study are more popular bitter-tasting foods in the Polish daily diet than other bitter-tasting items, such as aged cheddar cheese, red wine, arugula (rocket), or cilantro (coriander).

We observed that the $\mathrm{C}$ carriers of the TAS2R3 gene ate Brassica vegetables more frequently than the TT homozygotes (Table 4). There was also an interaction between the TAS2R 3 and TAS2R 5 genes and the simultaneous $\mathrm{C}$ carriers of $T A S 2 R 3$ and the $\mathrm{G}$ carriers of TAS2R5 ate Brassica vegetables more frequently than the TT/AA homozygotes. The higher CRP levels observed in the TT homozygotes of TAS $2 R 3$ could be partly explained by this lower frequency of Brassica vegetable intake (Table 3). Moreover, associations between the CRP level and the TT genotype of $T A S 2 R 3$ were also noted for subjects who selected all the bitter products studied less frequently (Table 4). Indole-3-carbinol from Brassica vegetables is a factor that prevents inflammation (Dinkova-Kostova, 2013), and it could thus be hypothesized that subjects who avoid Brassica vegetables in their diet may be more prone to inflammation. Serum total cholesterol and LDL cholesterol levels were also higher in the TT homozygotes than in the $\mathrm{C}$ carriers, who drank coffee or ate grapefruit less frequently. Together this suggests that the $T$ allele of the TAS $2 R 3$ gene may be associated with a lower intake of bitter food items rich in health-promoting compounds (Brassica vegetables, coffee and grapefruit), which in turn might also be associated with less favorable lipid profiles and higher CRP levels in serum.

The association analysis of rs2234012 (TAS2R5) genetic polymorphism and the frequency of the selected bitter products indicated that the $\mathrm{G}$ carriers chose grapefruit more frequently than the AA homozygotes (Table 3 ). When the study group was stratified by median intake frequency, those $\mathrm{G}$ carriers of the TAS2R5 genetic polymorphism who chose Brassica vegetables or grapefruit less frequently than the median value for the group proved to have significantly higher CRP serum levels than the AA homozygotes (Table 4). The associations between the frequency of intake of bitter products and the polymorphisms of TAS2R3 and TAS2R 5 remain unclear. It has previously been shown that simultaneous CC homozygotes of TAS $2 R 3$ and GG homozygotes of TAS $2 R 5$ reported less bitterness from sampled espresso coffee than the TT/AA homozygotes. Notably, this haplotype did not predict coffee liking or intake among the study cohort (Hayes et al., 2011). No association between these two polymorphisms and frequency of coffee intake was observed in our study. Interestingly, other researchers have shown that CC/GG homozygotes of TAS2R3 and TAS2R5 rate the greatest bitterness of ethanol compared to heterozygotes and TT/AA homozygotes (Nolden et al., 2016). Because the bitter ligands that activate TAS2R 3 and TAS2R 5 have been not identified yet, additional research is necessary to determine the reason for this discrepancy.

Although no ligand has been described for the TAS2R19 receptor (Meyerhof et al., 2010), the rs 10772420 polymorphism of the TAS2R19 gene has been studied in the context of variation in the bitterness of quinine intake (Hayes et al., 2015), as well as grapefruit (Hayes et al., 2011), coffee, tea and alcohol intake (Ong et al., 2018). It was reported that the 
A allele was associated with greater quinine bitterness, and AA homozygotes rated grapefruit juice as twice as bitter as GG homozygotes or heterozygotes. Moreover, the more sensitive A carriers liked grapefruit juice less than the less sensitive GG homozygotes (Hayes et al., 2011; 2015). Interestingly, A carriers drink less coffee compared with $\mathrm{G}$ carriers, but more tea and alcohol (Ong et al., 2018). In our study, there was no association between the frequency of bitter-tasting food intake and the rs 10772420 polymorphism. The results may be partly explained by linkage disequilibrium of the rs 10772420 polymorphism within TAS2R19 gene with other genetic polymorphisms located on chromosome 12 (i.e. TAS2R31), which were not measured in our study (Hayes et al., 2015) and small effect of the polymorphism studied (Ong et al., 2018). When the study group was stratified by median value of frequency intake, the AA homozygotes of TAS2R19 with a higher frequency of grapefruit intake had higher serum glucose levels than the G carriers (Table 4). The association between the TAS2R haplotype on human chromosome 12 with the regulation of glucose levels was already shown in a homogeneous Amish population (Dotson et al., 2008).

It has been already reported that the $\mathrm{C}$ allele of the TAS2R50 gene is associated with the decreased consumption of vegetables in general (Schembre et al., 2013), as well as of cilantro (Knaapila et al., 2012), and a higher risk of myocardial infarction (Shiffman et al., 2005), but we found no association between rs1376251 polymorphism within the TAS2R50 gene and the frequency of bitter food intake. When our study group was stratified by median frequency value of Brassica vegetables, coffee, or grapefruit intake, higher levels of TG, total cholesterol and LDL cholesterol were observed in the T carriers of rs 1376251 polymorphism (TAS2R50) than in the CC homozygotes for the group who chose Brassica vegetables more often than the median value, as well as for the group who chose grapefruit and coffee less often than the median value (Table 4). This means that T carriers of TAS2R 50 are more prone to the development of cardiovascular diseases, depending on the frequency of their eating particular bitter-tasting foods. As mentioned earlier, these foods contain different compounds which may affect the risk of cardiovascular diseases through various mechanisms, including upregulation of the mRNA levels of the enzymes involved in $\beta$-oxidation, such as peroxisome proliferator activated receptor (PPAR) $\alpha$, acyl-CoA oxidase, FA synthase in liver (Hirai et al., 2007), as well as inhibiting the production of TNF- $\alpha$ and MCP-1, which induce macrophage infiltration into adipose tissue cause inflammation (Chen et al., 2012) and arterial tissue remodeling. The differences between our results and previously reported outcomes (Shiffman et al., 2005) may be due to the different groups that were studied, different test models or an underpowered study.

Our study had also several limitations. The first is that a relatively small study group was used. Moreover, specific eating habits independent of taste are more pronounced in the elderly. Eating behavior is a complex trait that is affected by many factors (Chmurzynska and Mlodzik, 2017). Aversions to bitter food may occur from reasons other than bitter taste, including appearance, texture, social or cultural taboos associated with eating, but also from unpleasant gastrointestinal effects or food availability. Because eating habits are relatively stable attitudes towards food throughout life, they are difficult to change, especially in older age. On the other hand, the number of studies that focus on food choices in relation to gene polymorphism in the elderly is still very limited. The second limitation is the lack of additional sensory tests, so we could not directly observe bitterness perception and defined the subjects as taster or non-taster. The results of any one-tastant sensory tests should be carefully compared with real-life food intake, because food is a mixture of thousands of ligands that activate many taste receptors and other proteins involved in the taste sensation. Furthermore, the exclusion criteria did not involve drug treatments interfering with taste sensitivity. All the aforementioned factors could overshadow the influence of taste sensation on the frequency of bitter product intake.

We also realize that all nutrition assessments are fraught with different bias (Kipnis et al., 2003). The limitation of food frequency questionnaire (FFQ) method used in the study is that it only measures frequency of intake, without estimating portion size and calories in the whole diet. Because of the intake assessment method applied, several covariate factors, e.g. overall diet quality, were not included in the analyses. It should also be mentioned that the study 
group was enrolled originally for a different study that was performed previously (Chmurzynska et al., 2013). For this reason, the FFQ used for nutritional data collection was designed to assess folate intake and lacks some other bitter-tasting food items, i.e. cocoa and dark chocolate. Furthermore, the differences in bitter taste responses on sweet food selection, which can directly influence bitter food intake, were not studied here, but the aim of study was to investigate the connection between the frequency of bitter-tasting food intake, genetic polymorphism in selected TAS2Rs and blood biomarkers.

Our study shows that genetic polymorphisms may be associated with the frequency of eating a particular bitter food rich in glucosinolates or chlorogenic acid, which are known to protect against chronic disease development. Subjects with the T allele of TAS2R3 chose Brassica vegetables and those with the A allele of TAS2R 5 chose grapefruit less frequently than the opposite homozygotes. Genetic polymorphisms within TAS2R19 and TAS2R50 were not associated with the frequency of intake of the bitter-tasting food items studied. These polymorphisms were not associated with the frequency of coffee intake, as well as glucose level and lipid profile in serum. The TAS2R3 genotype was associated with CRP concentrations in blood, suggesting that frequent intake of bitter-tasting food may improve the CRP level in the serum. The SNPs of TAS2R5, TAS2R 19 and TAS2R50 were associated with blood biomarkers, depending on the frequency of eating particular foods (Table 4).

The results obtained in our study are difficult to interpret on the functional level, because TAS2R3, $T A S 2 R 19$ and TAS2R50 remain orphan receptors and for TAS2R5, only an artificial sweet-tasting agonist was described (Lossow et al., 2016). Two explanations could be possible. Firstly, genetic polymorphisms of taste receptors may influence metabolism indirectly by altering the frequency of intake of particular bitter-tasting food items, which contain metabolic active ingredients, i.e. isothiocyanates, caffeine, chlorogenic acid, naringin and naringenin (Alam et al., 2014; Choi et al., 2012; Dinkova-Kostova, 2013). Secondly, the bitter taste receptors, which are expressed also in extra-oral tissues, namely in the digestive tract (Avau et al., 2015) and autonomic nervous system (Tsuji et al., 2018), participate directly in controlling the release of gastrointestinal hormones, which modulate digestive behavior, satiety and body mass, as well as influencing metabolic biomarkers in blood.

The biological mechanisms of the associations identified require further investigation.

\section{REFERENCES}

Alam, M. A., Subhan, N., Rahman, M. M., Uddin, S. J., Reza, H. M., Sarker, S. D. (2014). Effect of citrus flavonoids, naringin and naringenin, on metabolic syndrome and their mechanisms of action. Adv. Nutr., 5, 404-417. https://doi.org/10.3945/an.113.005603

Avau, B., Rotondo, A., Thijs, T., Andrews, C. N., Janssen, P., Tack J., Depoortere, I. (2015). Targeting extra-oral bitter taste receptors modulates gastrointestinal motility with effects on satiation. Sci. Rep., 6, 15985. https://doi. org/10.1038/srep15985

Barragán, R., Coltell, O., Portolés, O., Asensio, E. M., Sorlí, J. V., Ortega-Azorín, C., ..., Corella, D. (2018). Bitter, sweet, salty, sour and umami taste perception decreases with age: sex-specific analysis, modulation by genetic variants and taste-preference associations in 18 to 80 year-old subjects. Nutrients, 18, E1539. https://doi. org/10.3390/nu10101539

Behrens, M., Foerster, S., Staehler, F., Raguse, J. D., Meyerhof, W. (2007). Gustatory expression pattern of the human TAS2R bitter receptor gene family reveals a heterogenous population of bitter responsive taste receptor cells. J. Neurosci., 27, 12630-12640. https://doi. org/10.1523/JNEUROSCI.1168-07.2007

Bufe, B., Hofmann, T., Krautwurst, D., Raguse, J.D., Meyerhof, W. (2002). The human TAS2R16 receptor mediates bitter taste in response to beta-glucopyranosides. Nat. Genet., 32, 397-401. https://doi.org/10.1038/ng1014

Chen, S., Ding, Y., Tao, W., Zhang, W., Liang, T., Liu, C. (2012). Naringenin inhibits TNF- $\alpha$ induced VSMC proliferation and migration via induction of HO-1. Food. Chem. Toxicol., 50, 3025-3031. https://doi.org/10.1016/ j.fct.2012.06.006

Chmurzynska, A., Mlodzik, M. A. (2017). Genetics of fat intake in the determination of body mass. Nutr. Res. Rev., 30, 106-117. https://doi.org/10.1017/S095442241 7000014

Chmurzynska, A., Malinowska, A. M., Twardowska-Rajewska, J., Gawecki, J. (2013). Elderly women: Homocysteine reduction by short-term folic acid supplementation resulting in increased glucose concentrations and affecting lipid metabolism (C677T MTHFR polymorphism). 
Nutrition, 29, 841-844. https://doi.org/10.1016/j.nut. 2012.09.015

Choi, Y., Kim, Y., Park, S., Lee, K. W., Park, T. (2012). Indole-3-carbinol prevents diet-induced obesity through modulation of multiple genes related to adipogenesis, thermogenesis or inflammation in the visceral adipose tissue of mice. J. Nutr. Biochem., 23, 1732-1739. https://doi.org/10.1016/j.jnutbio.2011.12.005

Chung, S., Domino, M. E., Stearns, S. C. (2009). The effect of retirement on weight. J. Gerontol. B: Psychol. Sci. Soc. Sci., 64, 656-665. https://doi.org/10.1093/geronb/ gbn044

Clinical Guidelines on the Identification, Evaluation, and Treatment of Overweight and Obesity in Adults. The Evidence Report. NHLBI Obesity Education Initiative Expert Panel on the Identification, Evaluation, and Treatment of Obesity in Adults (US). Bethesda (MD): National Heart, Lung, and Blood Institute. Report No. 98-4083 (1998).

Colares-Bento, F. C., Souza, V. C., Toledo, J. O., Moraes, C. F., Alho, C. S., Lima, R. M., ..., Nobrega, O. T. (2012). Implication of the G145C polymorphism (rs713598) of the TAS2r38 gene on food consumption by Brazilian older women. Arch. Gerontol. Geriatr., 54, e13-18. https://doi.org/10.1016/j.archger.2011.05.019

Dinkova-Kostova, A. T. (2013). Chemoprotection against cancer by isothiocyanates: A focus on the animal models and the protective mechanisms. Top. Curr. Chem., 329, 179-201. https://doi.org/10.1007/128_2012_337

Dotson, C. D., Zhang, L., Xu, H., Shin, Y. K., Vigues, S., Ott, S. H., ..., Munger, S. D. (2008). Bitter taste receptors influence glucose homeostasis. PLoS One, 3, e397. https://doi.org/10.1371/journal.pone.0003974

Duffy, V. B., Hayes, J. E., Davidson, A. C., Kidd, J. K., Kidd, K. K., Bartoshuk, L. M. (2010). Vegetable intake in college-aged adults is explained by oral sensory phenotypes and TAS2R38 genotype. Chemosens. Percept., 3, 137-148. https://doi.org/10.1007/s12078-010-9079-8

Hayes, J. E., Feeney, E. L., Nolden, A. A., McGeary, J. E. (2015). Quinine bitterness and grapefruit liking associate with allelic variants in TAS2R31. Chem. Senses, 40, 437-443. https://doi.org/10.1093/chemse/bjv027

Hayes, J. E., Wallace, M. R., Knopik, V. S., Herbstman, D. M., Bartoshuk, L. M., Duffy, V. B. (2011). Allelic variation in TAS2R bitter receptor genes associates with variation in sensations from and ingestive behaviors toward common bitter beverages in adults. Chem. Senses, 3, 311-319. https://doi.org/10.1093/chemse/bjq132

Hirai, S., Kim, Y.-II, Goto, T., Kang, M.-S., Yoshimura, M., Obata, A., ..., Kawada, T. (2007). Inhibitory effect of naringenin chalcone on inflammatory changes in the interaction between adipocytes and macrophages. Life Sci., 81, 1272-1279. https://doi.org/10.1016/j.lfs.2007. 09.001

Hong, E. P., Park, J. W. (2012). Sample size and statistical power calculation in genetic association studies. Genom. Inf., 10, 17-122. https://doi.org/10.5808/GI. 2012.10.2.117

Kim, U., Wooding, S., Ricci, D., Jorde, L. B., Drayna, D. (2005). Worldwide haplotype diversity and coding sequence variation at human bitter taste receptor loci. Hum. Mutat., 26, 3, 199-204. https://doi.org/10.1002/ humu. 20203

Kipnis, V., Subar, A. F., Midthune, D., Freedman, L. S., Ballard-Barbash, R., Troiano, R. P., ..., Carroll, R. J. (2003). Structure of dietary measurement error: results of the OPEN biomarker study. Am. J. Epidemiol., 1, 14-21. https://doi.org/10.1093/aje/kwg091

Knaapila, A., Hwang, L. D., Lysenko, A., Duke, F. F., Fesi, B., ..., Reed, D. R. (2012). Genetic analysis of chemosensory traits in human twins. Chem. Senses, 37, 869-881. https://doi.org/10.1093/chemse/bjs070

Kourouniotis, S., Keast, R. S., Riddell, L. J., Lacy, K., Thorpe, M. G., Cicerale, S. (2016). The importance of taste on dietary choice, behavior and intake in a group of young adults. Appetite, 103, 1-7. https://doi.org/10.1016/j.appet.2016.03.015

Kraehenbuehl, K., Page-Zoerkler, N., Mauroux, O., Gartenmann, K., Blank, I., Bel-Rhlid, R. (2017). Selective enzymatic hydrolysis of chlorogenic acid lactones in a model system and in a coffee extract. Application to reduction of coffee bitterness. Food Chem., 218, 9-14. https://doi.org/10.1016/j.foodchem.2016.09.055

Lossow, K., Hübner, S., Roudnitzky, N., Slack, J. P., Pollastro, F., Behrens, M., Meyerhof, W. (2016). Comprehensive analysis of mouse bitter taste receptors reveals different molecular receptive ranges for orthologous receptors in mice and humans. J. Biol. Chem., 291, 15358 15377. https://doi.org/10.1074/jbc.M116.718544

McClave, J. T., Sincich, T. (2003). Statistics, 9th ed. New Jersey: Prentice.

Meyerhof, W., Batram, C., Kuhn, C., Brockhoff, A., Chudoba, E., Bufe, B., ..., Behrens, M. (2010). The molecular receptive ranges of human TAS2R bitter taste receptors. Chem. Senses, 35, 157-170. https://doi.org/10.1093/ chemse/bjp092

Mikołajczyk-Stecyna, J., Malinowska, A. M., Chmurzynska, A. (2017). TAS2R38 and CA6 genetic polymorphisms, frequency of bitter food intake, and blood biomarkers 
among elderly woman. Appetite, 116, 57-64. https://doi. org/10.1016/j.appet.2017.04.029

Nolden, A. A., McGeary, J. E., Hayes, J. E. (2016). Differential bitterness in capsaicin, piperine, and ethanol associates with polymorphisms in multiple bitter taste receptor genes. Physiol. Behav., 156, 117-127. https:// doi.org/10.1016/j.physbeh.2016.01.017

Ogawa, T., Annear, M. J., Ikebe, K., Maeda, Y. (2017). Taste-related sensations in old age. J. Oral Rehabil., 44, 626-635. https://doi.org/10.1111/joor.12502

Ong, J. S., Hwang, D. L., Zhong, V. W., An, J., Gharahkhani, P., Breslin, P. A. S., ..., Cornelis, M. C. (2018). Understanding the role of bitter taste perception in coffee, tea and alcohol consumption through Mendelian randomization. Sci. Rep., 15, 16414. https://doi.org/10.1038/ s41598-018-34713-z

Rabin, B. A., Boehmer, T. K., Brownson, R. C. (2007). Cross-national comparison of environmental and policy correlates of obesity in Europe. Eur. J. Public Health, 17, 53-61. https://doi.org/10.1093/eurpub/ck1073

Sacerdote, C., Guarrera, S., Smith, G. D., Grioni, S., Krogh, V., Masala, G., ..., Vineis, P. (2007). Lactase persistence and bitter taste response: instrumental variables and mendelian randomization in epidemiologic studies of dietary factors and cancer risk. Am. J. Epidemiol., 166, 576-581. https://doi.org/10.1093/aje/kwm113

Schembre, S. M., Cheng, I., Wilkens, L. R., Albright, C. L., Marchand, le L. (2013). Variations in bitter-taste receptor genes, dietary intake, and colorectal adenoma risk. Nutr. Cancer, 65, 982-990. https://doi.org/10.1080/016 35581.2013 .807934
Shiffman, D., Ellis, S. G., Rowland, C. M., Malloy, M. J., Luke, M. M., Iakoubova, O. A., ..., Kane, J. P. (2005). Identification of four gene variants associated with myocardial infarction. Am. J. Hum. Genet., 77, 596-605. https://doi.org/10.1086/491674

Third Report of the National Cholesterol Education Program (NCEP). Expert Panel on Detection, Evaluation, and Treatment of High Blood Cholesterol in Adults (Adult Treatment Panel III). Final report. NIH Publication No. 02-5215 (2002). Circulation, 106, 3143-421.

Toth, M. J., Tchernof, A., Sites, C. K., Poehlman, E. T. (2000). Menopause-related changes in body fat distribution. Ann. NY Acad. Sci., 904, 502-506. https://doi. org/10.1111/j.1749-6632.2000.tb06506.x

Tsuji, T., Tanaka, S., Bakhshishayan, S., Kogo, M., Yamamoto, T. (2018). Olfactory stimulation modulates the blood glucose level in rats. Int. J. Med. Sci., 15, 269273. https://doi.org/10.7150/ijms. 21528

Vasilopoulos, T., Morey, T. E., Dhatariya, K., Rice, M. J. (2016). Limitations of significance testing in clinical research: A review of multiple comparison corrections and effect size calculations with correlated measures. Anesth. Analg., 122, 825-30. https://doi.org/10.1213/ ANE.0000000000001107

Yeh, E. T. H., Willerson, J. T. (2003). The coming of age of C-reactive protein: using inflammation markers in cardiology. Circulation, 107, 370-372. https://doi. org/10.1161/01.cir.0000053731.05365.5a 